\title{
1 Active modulation of human erythrocyte mechanics
}

2 Lennart Kuck ${ }^{1}$, Jason N. Peart ${ }^{2}$, Michael J. Simmonds ${ }^{1}$

$3 \quad{ }^{1}$ Biorheology Research Laboratory, Griffith University Gold Coast, Queensland, Australia

$4 \quad{ }^{2}$ School of Medical Science, Griffith University Gold Coast, Queensland, Australia

10 Corresponding author

11 Michael J. Simmonds, PhD

12 Biorheology Research Laboratory, Griffith University, Gold Coast, Australia

13 Tel: $\quad+61(7) 55528529$

14 Fax: $+61(7) 55528674$

15 Email: mike@thesimmonds.id.au 
21 The classic view of the red blood cell (RBC) presents a biologically inert cell, that upon

22 maturation, has limited capacity to alter its physical properties. This view developed largely

23 due to the absence of translational machinery and inability to synthesise or repair proteins in

24 circulating RBC. Recent developments have challenged this perspective, in light of

25 observations supporting the importance of post-translational modifications, and greater

26 understanding of ion movement in these cells, that each regulate a myriad of cellular

27 properties. There is thus now sufficient evidence to induce a step-change in understanding of

28 RBC; rather than passively responding to the surrounding environment, these cells have the

29 capacity to actively regulate their physical properties and thus alter flow behaviour of blood.

30 Specific evidence supports that the physical and rheological properties of RBC are subject to

31 active modulation, primarily by the second-messenger molecules nitric oxide (NO) and

32 calcium-ions $\left(\mathrm{Ca}^{2+}\right)$. Further, an isoform of nitric oxide synthase is expressed in RBC (RBC-

33 NOS), which has been recently demonstrated to have an active role in regulating the physical

34 properties of RBC. Mechanical stimulation of the cell membrane activates RBC-NOS leading

35 to NO generation, which has several intracellular effects, including the S-nitrosylation of

36 integral membrane components. Intracellular concentration of $\mathrm{Ca}^{2+}$ is increased upon

37 mechanical stimulation via the recently identified mechano-sensitive cation channel, piezo1.

38 Increased intracellular $\mathrm{Ca}^{2+}$ modifies the physical properties of $\mathrm{RBC}$ by regulating cell

39 volume and potentially altering several important intracellular proteins. A synthesis of recent

40 advances in understanding of molecular processes within RBC thus challenges the classic

41 view of these cells, and rather indicates a highly active cell with self-regulated mechanical 42 properties. 


\section{Role of cell mechanics in oxygen delivery}

46 Cellular respiration is vital to sustained metabolism, and therefore life, and is only possible

47 due to the integration of the cardiovascular, pulmonary, and circulatory systems. One of the

48 challenges to oxygen loading, and subsequent transport, within the circulatory system is the

49 extremely small apertures that blood needs to navigate: the smallest capillaries $(2-3 \mu \mathrm{m})$ in

50 the pulmonary and peripheral circulations are a fraction of the resting diameter $(\sim 8 \mu \mathrm{m})$ of

51 red blood cells (RBC); consequently, the RBC is morphologically agile, with a unique ability

52 among all mammalian cells to change shape and subsequently return to its resting state

53 without any appreciable negative effects (e.g., hysteresis; 10). A pathologically reduced

54 ability of RBC to deform has thus long been considered a precursor for heightened risk of

55 tissue ischaemia at both micro- and macro-vascular scales, given rigid cells: i. have limited

56 capability to perfuse the rich microvascular network of organs (47); and, ii. affect the bulk

57 properties of blood through increasing the resistance to flow by contributing to hyperviscosity

58 (4). Indeed, even the capacity for oxygen dissociation from haemoglobin during tissue

59 perfusion has been reported to be limited by decreased cellular deformability (56). Further,

60 many disease states associated with tissue necrosis, ischaemia, and/or hypoxia are

61 characterised by poor cell mechanics of RBC (53). Thus, despite a vital role for optimal

62 cellular mechanics being well established, the molecular regulation of these physical

63 properties is only recently emerging.

64

65 Classic physical properties enable erythrocyte deformability 
66 The properties of RBC that were classically recognised to confer their unique capacity to

67 deform in response to mechanical stimulation included the excess surface area relative to cell

68 volume, the intracellular viscosity, and a visco-elastic cellular membrane (10). The excessive

69 surface area of RBC is clearly observed when the cell is compared with a perfect sphere: while

70 the average volume of an $\mathrm{RBC}\left(\sim 90 \mu \mathrm{m}^{3}\right)$ could be contained in a sphere with only $97 \mu \mathrm{m}^{2}$ of

71 surface area, the surface area of an $\mathrm{RBC}$ is $\sim 44 \%$ larger $\left(\sim 140 \mu \mathrm{m}^{2}\right)$ and thus yields a surface

72 area-to-volume ratio that approaches $1.5(10)$. The resultant excess surface area is provided by

73 the bi-concave structure of the RBC membrane and facilitates significantly greater capacity to

74 deform than a spherical shape. The intracellular viscosity of RBC is largely determined by

75 haemoglobin concentration suspended in the cytosol. The mean intracellular viscosity of

$76 \mathrm{RBC}$ is $\sim 7 \mathrm{mPa} \cdot \mathrm{s}$ at normal haemoglobin content, which is comparatively higher than the

77 viscosity of the surrounding plasma $(\sim 1.2 \mathrm{mPa} \cdot \mathrm{s} ; 10)$. This disparity contributes to the

78 deformation of RBC by facilitating an independent rotation of the cellular membrane around

79 the cytoplasm, termed tank-treading (16). While the earliest interpretation of tank-treading

80 indicated that the cell membrane was mostly independent of internal structures (16), advances

81 over the last decades support that various entities complex with the intracellular surfaces of

82 the cell membrane, which indeed may even serve to enhance the mechanical properties of the

83 cell (36).

84 Further, the exceptionally deformable capacity of the RBC membrane has been long

85 observed; this cell provides the template for lipid bilayer models of cell membranes and is

86 noted for its exceptional capacity to deform upon exposure to shearing forces, subsequently

87 reassuming its original bi-concave shape (4). The RBC membrane contains a cytoskeletal

88 mesh-network with integral proteins establishing connections with the lipid bilayer, which

89 stabilises the characteristic bi-concave shape of RBC. Collectively, the properties that permit

90 a remarkable capacity of $\mathrm{RBC}$ to deform have been studied (Fig. 1), and until recently the 
91 determinants of these properties were thought to be static. Recent advances challenge this

92 perspective and support an 'active' regulation of mechanical properties that may acutely alter

93 the physical properties of RBC.

95 The molecular structure of the erythrocyte membrane facilitates cellular deformability

96 The molecular composition of both the cytoskeletal and lipid bilayer components of RBC has

97 been subject of extensive study during the past century $(21,25)$. The cytoskeleton consists of

98 spectrin filaments (i.e., $\alpha$ - and $\beta$-subunits, which are antiparallel isomers), assembled in

99 conjunction with filamentous actin to form hetero-tetramers in head-to-head manner (Fig. 2).

100 These filaments are interconnected to the lipid bilayer: i. via complexation with integral

101 membrane proteins band 3 and glycophorin; or ii. via actin and protein 4.1 (52). Smith et al.,

102 (49) recently demonstrated that the spectrin-actin cytoskeleton is stabilised by an isoform of

103 non-muscular filamentous myosin (NMIIA). NMIIA is a hexameric actin-binding ATPase

104 comprised of two heavy chains, two light chains, and two regulatory chains, and importantly

105 is capable of contraction - which in other cell lines has been shown to be vitally important to

106 cell function (46). Knowledge around the role that NMIIA exerts within RBC is still

107 developing, although it has been recently demonstrated that via linkage to actin in the

108 cytoskeletal component of the membrane, tension is regulated to maintain the cell's unique

109 bi-concave shape (49). It follows that the mechanical properties that are promoted by this

110 unique shape are thus also integrally affected by NMIIA (49). Indeed, when NMIIA is

111 inhibited using a NMIIA-specific inhibitor (i.e., blebbistatin), RBC exhibit elongated

112 morphology at rest and lose their membrane curvature (49). How phosphorylation or other

113 post-translational modifications may alter NMIIA-contractility and RBC mechanics, 
114 however, remains to be resolved, although it is a valid area of interest given observations in

115 other cell lines $(9,46,58)$

116 Collectively, although the molecular identity and structure of the RBC membrane has been

117 largely determined, the interactive functions of numerous proteins remain unresolved, as is

118 the dynamic re-assembly of membrane components during cellular deformation. Moreover,

119 while classically the physical properties of RBC were viewed as the singular determinants of

120 their extensive deformability, post-translational modifications of membrane proteins in

121 particular have been suggested as mechanisms to acutely alter RBC mechanics $(13,24,32)$.

123 Red blood cells are not sedentary: the role of nitrogen species

124 The concept that the physical properties of RBC may be modulated "actively" - that is, 125 dynamically altered upon exposure to mechanotransductive stimuli - has emerged only over

126 the past decade or so. While the presence of a process responsible for generating intracellular

127 NO in RBC was speculated for some time (38), Kleinbongard et al., (31) only relatively 128 recently employed independent and complimentary methods to demonstrate that RBC contain 129 a NOS isoform that was distinct to the neuronal and inducible forms. Indeed, several key 130 physical features were shown to reflect those of the endothelial-type NOS. The activity of 131 this so-called "RBC-NOS" isoform was shown to be dependent on the substrate L-arginine, 132 was calcium-sensitive, and also sensitive to phosphorylation via the PI3-kinase pathway (31) 133 - all properties shared with endothelial NOS. Various groups subsequently confirmed that 134 RBC carry a functional NOS-type enzyme, which facilitates endogenous production of nitric 135 oxide $(24,39,51,54)$. Elucidating potential activation and down-stream targets mechanisms 136 of RBC-NOS was thus of interest. Shear stress - induced either in vitro by exposing RBC to 137 fluid shear in a microchannel or in vivo by increasing blood flow through exercise - may act 
138 as an activator of RBC-NOS by inducing phosphorylation at its active residue serine 1177

$139\left(\operatorname{ser}^{1177}\right)(51,54)$. Increased phosphorylation of this residue has been reported by various 140 groups to be closely associated with increased RBC deformability (22, 24, 32), while 141 inhibition of RBC-NOS was previously shown to be associated with impaired cell 142 deformability (24). The concrete activation mechanism of RBC-NOS is currently inferred 143 from its alleged structural twin - endothelial NOS - although requires direct evidence. Given $144 \mathrm{RBC}$ and endothelial cells differ significantly in structure and intracellular composition (i.e., 145 RBC lack a nucleus and Golgi apparatus, which are pivotal in activation of endothelial NOS; 146 44), further elucidation is required to examine the RBC-NOS isoform to discern its molecular 147 mechanisms in higher resolution.

148 Activation mechanism and down-stream targets of erythrocyte-derived nitric oxide synthase

149 The primary mechanism currently proposed to elicit activation and mobilisation of RBC150 NOS is mechanical stimulation $(13,54)$; that is, it is thought that physical forces deforming 151 the cell membrane may be transduced into biochemical events that stimulate NO-generation 152 directly, or alternatively via an independent method involving $\mathrm{Ca}^{2+}$-signalling.

153 Investigations aimed at discerning the effects of varying shear stress magnitudes on RBC 154 deformability and NO-metabolism in flow led to a dose-response relationship being observed 155 between shear stress and NO-production (27). Moreover, in vitro application of shear stress 156 in the physiological range during laminar flow (i.e., comparable to forces present in the 157 human circulatory system) was shown to elicit phosphorylation of RBC-NOS ser ${ }^{1177}$, which 158 occurred in concert with increased cell deformability (32). These data support that shear 159 stress appears to activate RBC-NOS, and lead to predictable increases in intracellular NO 160 production, that may impact cellular deformability. The mechanism for the shear-induced 161 RBC-NOS activation remains elusive, although Ulker et al., (54) demonstrated that while 162 shear exposure increased the phosphorylation of RBC-NOS at $\operatorname{ser}^{1177}$, intracellular 
163 concentrations of $\mathrm{NO}$ and intracellular $\mathrm{Ca}^{2+}$ concentration increased in tandem. Grau et al.,

164 (24) independently, demonstrated that NO produced during mechanical stimulation may

165 reversibly incorporate into the cytoskeletal component of the cell membrane, as evidenced by

166 increased S-nitrosylation of integral membrane proteins. Specifically, Grau et al (24)

167 suggested that NO appears to bind the exposed sulphur atoms in $\alpha$ - and $\beta$-spectrin filaments

168 forming nitrosylated residues, and they hypothesised that this process may explain the

169 increased cellular deformability in the presence of increased intracellular NO concentration.

170 Further observations to confirm the S-nitrosylation of the cell membrane remain elusive,

171 although provide a tantalising suggestion of a mechanism for shear-mediated modulation of

172 cell deformability with implications in vascular perfusion.

173 It collectively appears that shear stress activates RBC-NOS, and thus also increases

174 intracellular availability of $\mathrm{NO}$, which may impact the physical properties of the cell

175 membrane via S-nitrosylation. How the flux of $\mathrm{Ca}^{2+}$ during mechanical stimulation of the cell

176 membrane - such as when RBC pass narrow capillaries to facilitate gas exchange - is related

177 to these events remains a topic of current investigation. While these characteristics infer

178 striking similarities between endothelial NOS and RBC-NOS, it is stressed that these cells

179 maintain fundamental biological differences, no less than the obvious differences in intracellular

180 organelles. Several groups are thus currently exploring the function of RBC-NOS $(24,32,54)$,

181 although fundamental studies into the structure of RBC-NOS, and its potential localisation within

182 RBC are ongoing, yet stand to deliver much greater understanding on the regulatory role of this 183 protein.

185 Modulation of erythrocyte mechanics by intracellular calcium 
186 Incubation of RBC with calcium was classically reported to induce membrane rigidification, 187 and thus decreased cellular deformability; at least for experiments employing high 188 concentrations of extracellular $\mathrm{Ca}^{2+}(45)$. The mechanism of cellular rigidification, however, 189 has never been well understood, leading to inferences of a hypothetical ionic association of $190 \mathrm{Ca}^{2+}$ with some membrane components (41). It is clear that intracellular calcium of $\mathrm{RBC}$ is 191 maintained at a comparatively low concentration $(\sim 40-60 \mathrm{nmol} \cdot \mathrm{L}-1 ; 6)$ relative to that of the 192 surrounding plasma $(\sim 1.8 \mathrm{mmol} \cdot \mathrm{L}-1 ; 34)$, owing to the action of powerful calcium-ATPases 193 (PMCA; 42). Given RBC do not possess mitochondria, and glycolysis is the sole pathway of 194 endogenous ATP-generation, the capacity of RBC to maintain such a tightly regulated 195 intracellular calcium concentration is thus dependent on the metabolic status of the cell.

196 O'Rear and colleagues (41) hypothesised calcium may impair the flexibility of the cell 197 membrane due to electrostatic interaction of this cation with the membrane lipid bilayer - at 198 least in the context of RBC being exposed to supraphysiological shears. Calcium depletes 199 cellular ATP (35), which was initially thought to be essential for maintaining RBC 200 deformability (57), by forcing high PMCA-activity. On the other hand, Clark et al., (11) 201 demonstrated that ATP-depletion and calcium-accumulation have distinct effects on cellular 202 deformability, hypothesising that the primary consequence of increased intracellular calcium 203 is activation of the Gárdos channel (for a review, see 37) and subsequent loss of intracellular 204 fluid. Briefly, the Gárdos channel is a calcium-activated potassium channel embedded in the 205 RBC membrane (19) that facilitates transport of potassium-ions $\left(\mathrm{K}^{+}\right)$to the extracellular 206 space (33), and thus also water according to an osmotic shift, leading to a decrease in cell 207 volume (i.e., Gárdos effect; Fig. 3). Of note, while the intracellular fluid leaving the RBC 208 contains chloride ions $\left(\mathrm{Cl}^{-}\right)$and water, haemoglobin is unable to leave the cell via this 209 mechanism. It follows that intracellular viscosity increases due to the resultant higher relative 210 abundance of haemoglobin, which has been experimentally validated and suggested to induce 
211 impaired cellular mechanics (55). Recent developments have led to a paradigm shift

212 indicating that the Gárdos effect may have valuable contributions to the maintenance of

213 cellular mechanics, at least when shear stresses and/or calcium flux is maintained within

214 physiological limits.

217 Early patch-clamp experiments performed on RBC to examine stretch-activated currents

218 found evidence of a transient $\mathrm{Ca}^{2+}$-flux that occurred in immediate response to mechanical

219 stimulation. A negative pressure was placed onto single RBC using micropipettes to induce a

220 reproducible deformation of the cell while isolating a membrane patch to record currents

221 (14). It was observed that secondary to the $\mathrm{Ca}^{2+}$-trace, $\mathrm{K}^{+}$was transported in the opposite 222 direction across the cell membrane (14). The authors thus hypothesised a possible interplay 223 between a mechanically-activated calcium channel (that was yet to be identified) and the 224 calcium-activated potassium channel, termed the Gárdos channel. Subsequent investigations 225 into stretch-activated ion channels in the RBC membrane became more complicated given 226 that the gold standard method - patch clamping - itself involves applying a mechnical 227 stimulus to the membrane which could evoke currents from mechanically-activated channels 228 (for a review, see 29).

229 Danielczok and colleagues (13) used in vivo and in vitro methods to assess transient calcium230 increases within deformed $\mathrm{RBC}$, visualised using a fluorescent probe. Upon cellular 231 deformation of the RBC membrane, intracellular calcium transiently increased (13). While 232 the RBC membrane is highly permeable to anions, it is poorly permeable to cations, and thus 233 calcium flux involves transporters/pumps residing within the membrane. The hundreds of 234 both selective, and non-selective transports are now known, with the vast majority of these 
235 routes being voltage sensitive (for a review, see 2). Danielczok and colleagues (13) thus 236 examined whether mechanosensitive cation-channels (i.e., piezo1), shown to be expressed on

237 the RBC membrane (8), were critically involved in the transient calcium-response.

238 Application of a specific piezo1-blocker, the peptide GsMTx-4 (1), abolished the expected

239 flux in calcium-content caused by stretching of the RBC membrane. Rheological and

240 biophysical measurements to assess the contribution of this mechanism to tissue perfusion

241 more globally (e.g., quantification of flow velocities, shear stresses, transit times), however,

242 were not performed in these experiments and would present a valuable addition to the

243 observations of Danielczok and colleagues (13). Moreover, quantifying the alterations in cell

244 volume as RBC pass narrow capillaries is required, in conjunction with investigations aimed

245 to discern the reversibility of this phenomenon both on a cellular (e.g., return to normal cell

246 size) and molecular level (e.g., ion-flux during states of reduced cell volume that facilitate a

247 restoration of cellular homeostasis). Integrative studies investigating the interplay between

248 mechanically-activated NO-generation and $\mathrm{Ca}^{2+}$-signalling pathways present ambitious albeit

249 valuable directions for full understanding of the RBC active regulation of its mechanical

250 properties. Given the calcium-dependency for activation of NOS observed in endothelial cells

251 (17), in addition to existing evidence supporting a similar mechanism in the RBC $(31,54)$, it

252 is plausible to suspect that cross-talk between these two signalling molecules exists.

Relevance in mechanical circulatory support and blood disorders

The mechanics of RBC are known to be impaired in a vast variety of pathologies (e.g., type 2

256 diabetes, coronary artery disease; 48, 53) which manifest in circulatory defects. Further, 257 given these diseases may progress to require interventions facilitated by mechanical 258 circulatory support (e.g., cardiopulmonary bypass; ventricular assist devices), which exert 
259 supraphysiological mechanical force on the blood (18), alterations in the sensitivity of 260 second-messenger pathways may be particularly implicated in commonly observed post261 surgical complications (30). Impaired transduction of $\mathrm{NO}-$ and $\mathrm{Ca}^{2+}$-regulated signalling 262 contributing to the observed rheological impairments in clinical conditions and after high263 shear exposure presents a therapeutic opportunity. Stimulating or inhibiting mechanically264 activated signalling pathways by pharmacological means could ameliorate the observed 265 deficiencies in sustaining oxygen-delivery.

266 Sickle cell anaemia is caused by a single point mutation in exon I coding for beta-globin (12)

267 which results in polymerisation of the haemoglobin beta-chain and thus 'sickling' of RBC 268 under local hypoxia. Repeated collapses of RBC - such as through sickling when traversing 269 the venous circulation - would provide a mechanical stimulus even stronger than shrinking 270 induced by exposure to an hypotonic solution, which has been independently shown to induce 271 piezo-1 activation and calcium-influx (13). The Gárdos effect has been associated with sickle 272 cell pathology (i.e., exacerbating the 'sickling'; 28) and inhibitors of the Gárdos channel 273 (e.g., clotrimazole and senicapoc) have been therapeutically explored, although these agents 274 proved ineffective in reducing vascular crisis events despite decreasing the relative 275 abundance of 'sickled' cells and haemolysis (7, 50). Homozygous piezo1-mutations are 276 expressed in $20 \%$ of the sickle cell anaemia population in contrast to $5 \%$ in the general 277 population (40); however, preliminary clinical observations of piezo1-mutations in sickle cell 278 anaemia patients did not significantly associate with disease severity (43). Independent 279 studies established abnormal NO-metabolism in sickle cell anaemia patients; that is, sickle 280 RBC present with increased NOS-activation and NO-production which are not associated 281 with improved rheological characteristics (23). It appears, however, that NO may inhibit the 282 Gárdos channel through interaction with cysteine residues on the extracellular side of the 283 RBC membrane, thereby ameliorating the Gárdos effect $(3,5)$. Alternatively, ionic volume- 
284 regulation via the Gárdos effect could offset NO-dependent increases in membrane

285 flexibility; however, further studies investigating the concrete interplay between these 286 pathways are required.

287 Mutations in the genes coding for piezol and the Gárdos channel have been reported in 288 patients suffering from xerocytosis and other forms of rare anaemia $(15,20)$. The rheological 289 properties and calcium-homeostasis of RBC were significantly altered, providing a potential 290 mechanistic explanation for the premature haemolysis observed in these patients (26).

291 Collectively, it appears that $\mathrm{RBC}$-volume regulation via $\mathrm{Ca}^{2+}$-homeostasis and endogenous 292 NO-generation play pivotal roles in classic blood disorders rooted in abnormal properties of 293 RBC. Given that only symptomatic treatments currently exist for these genetic blood 294 disorders, pharmacological interventions to correct the acute regulation of $\mathrm{Ca}^{2+}$ and $\mathrm{NO}$ 295 within $\mathrm{RBC}$ is a promising approach to restore cellular mechanics and ensure tissue 296 perfusion. For example, specific inhibitors of piezo1-channels (e.g., GsMTx4) may be titrated 297 to prevent intracellular $\mathrm{Ca}^{2+}$-overload, while stimulators of RBC-NOS (e.g., L-arginine) or 298 up-stream kinases (e.g., 740 Y-P) could increase RBC-derived NO and thus improve cell 299 mechanics which ultimately increase tissue perfusion.

\section{Conclusion}

302 Evidence has accumulated over the past decade establishing a significant role for second303 messenger molecules, such as $\mathrm{NO}$ and $\mathrm{Ca}^{2+}$, in contributing to the active regulation of the 304 physical properties of RBC. While several interactions and mechanisms remain to be 305 elucidated, it appears that tight control over the concentration of these messengers is pivotal 306 for ensuring RBC homeostasis, cellular deformability, and ultimately tissue perfusion. Thus 307 while the last decade has provided a new perspective of the active regulation of $\mathrm{RBC}$, 308 integration of molecular techniques, electrophysiology, and rheological assessment of blood 
appears a fruitful avenue for improved therapeutic approaches for cardiometabolic and

310 haematologic disorders, and further in the refinement of mechanical circulatory support

311 devices that remain plagued by poor complication rates.

\section{References}

313 1. Bae C, Sachs F, and Gottlieb PA. The mechanosensitive ion channel Piezo1 is 314 inhibited by the peptide GsMTx4. Biochemistry 50: 6295-6300, 2011.

315 2. Balach MM, Casale CH, and Campetelli AN. Erythrocyte plasma membrane 316 potential: past and current methods for its measurement. Biophys Rev 11: 995-1005, 2019.

317 3. Barodka V, Mohanty JG, Mustafa AK, Santhanam L, Nyhan A, Bhunia AK, 318 Sikka G, Nyhan D, Berkowitz DE, and Rifkind JM. Nitroprusside inhibits calcium319 induced impairment of red blood cell deformability. Transfusion 54: 434-444, 2014.

320 4. Baskurt OK, and Meiselman HJ. Blood rheology and hemodynamics. Semin 321 Thromb Hemost 29: 435-450, 2003.

322 5. Belanger AM, Keggi C, Kanias T, Gladwin MT, and Kim-Shapiro DB. Effects of 323 nitric oxide and its congeners on sickle red blood cell deformability. Transfusion 55: 2464$3242472,2015$.

325 6. Bogdanova A, Makhro A, Wang J, Lipp P, and Kaestner L. Calcium in red blood 326 cells-a perilous balance. International journal of molecular sciences 14: 9848-9872, 2013.

327 7. Brugnara C, Gee B, Armsby CC, Kurth S, Sakamoto M, Rifai N, Alper SL, and 328 Platt OS. Therapy with oral clotrimazole induces inhibition of the Gardos channel and 329 reduction of erythrocyte dehydration in patients with sickle cell disease. J Clin Invest 97: 330 1227-1234, 1996.

$331 \quad$ 8. Cahalan SM, Lukacs V, Ranade SS, Chien S, Bandell M, and Patapoutian A. 332 Piezo1 links mechanical forces to red blood cell volume. eLife 4: 2015.

333 9. Cai Y, Rossier O, Gauthier NC, Biais N, Fardin MA, Zhang X, Miller LW, 334 Ladoux B, Cornish VW, and Sheetz MP. Cytoskeletal coherence requires myosin-IIA 335 contractility. J Cell Sci 123: 413-423, 2010.

336 10. Chien S. Red cell deformability and its relevance to blood flow. Annual review of 337 physiology 49: 177-192, 1987.

338 11. Clark MR, Mohandas N, Feo C, Jacobs MS, and Shohet SB. Separate mechanisms 339 of deformability loss in ATP-depleted and Ca-loaded erythrocytes. J Clin Invest 67: 531-539, 3401981.

341 12. Connes P, Alexy T, Detterich J, Romana M, Hardy-Dessources MD, and Ballas 342 SK. The role of blood rheology in sickle cell disease. Blood Rev 30: 111-118, 2016.

343 13. Danielczok JG, Terriac E, Hertz L, Petkova-Kirova P, Lautenschlager F, 344 Laschke MW, and Kaestner L. Red Blood Cell Passage of Small Capillaries Is Associated 345 with Transient $\mathrm{Ca}(2+)$-mediated Adaptations. Frontiers in physiology 8: 979, 2017.

346 14. Dyrda A, Cytlak U, Ciuraszkiewicz A, Lipinska A, Cueff A, Bouyer G, Egee S, 347 Bennekou P, Lew VL, and Thomas SL. Local membrane deformations activate Ca2+348 dependent $\mathrm{K}+$ and anionic currents in intact human red blood cells. PloS one 5: e9447, 2010.

349 15. Fermo E, Bogdanova A, Petkova-Kirova P, Zaninoni A, Marcello AP, Makhro 350 A, Hanggi P, Hertz L, Danielczok J, Vercellati C, Mirra N, Zanella A, Cortelezzi A, 351 Barcellini W, Kaestner L, and Bianchi P. 'Gardos Channelopathy': a variant of hereditary 352 Stomatocytosis with complex molecular regulation. Sci Rep 7: 1744, 2017. 
353 16. Fischer TM, Stohr-Lissen M, and Schmid-Schonbein H. The red cell as a fluid

354 droplet: tank tread-like motion of the human erythrocyte membrane in shear flow. Science 355 202: 894-896, 1978.

356 17. Forstermann U, Pollock JS, Schmidt HH, Heller M, and Murad F. Calmodulin357 dependent endothelium-derived relaxing factor/nitric oxide synthase activity is present in the 358 particulate and cytosolic fractions of bovine aortic endothelial cells. Proc Natl Acad Sci U S 359 A 88: 1788-1792, 1991.

360 18. Fraser KH, Zhang T, Taskin ME, Griffith BP, and Wu ZJ. A quantitative comparison of mechanical blood damage parameters in rotary ventricular assist devices: shear stress, exposure time and hemolysis index. Journal of biomechanical engineering 134: $081002,2012$.

19. Gardos G. The function of calcium in the potassium permeability of human erythrocytes. Biochimica et biophysica acta 30: 653-654, 1958.

20. Glogowska E, Schneider ER, Maksimova Y, Schulz VP, Lezon-Geyda K, Wu J, Radhakrishnan K, Keel SB, Mahoney D, Freidmann AM, Altura RA, Gracheva EO, Bagriantsev SN, Kalfa TA, and Gallagher PG. Novel mechanisms of PIEZO1 dysfunction in hereditary xerocytosis. Blood 130: 1845-1856, 2017.

21. Gorter E, and Grendel F. On Bimolecular Layers of Lipoids on the Chromocytes of the Blood. J Exp Med 41: 439-443, 1925.

22. Grau M, Friederichs P, Krehan S, Koliamitra C, Suhr F, and Bloch W. Decrease in red blood cell deformability is associated with a reduction in RBC-NOS activation during storage. Clinical hemorheology and microcirculation 60: 215-229, 2015.

23. Grau M, Mozar A, Charlot K, Lamarre Y, Weyel L, Suhr F, Collins B, Jumet S, Hardy-Dessources MD, Romana M, Lemonne N, Etienne-Julan M, Antoine-Jonville S, Bloch W, and Connes P. High red blood cell nitric oxide synthase activation is not associated with improved vascular function and red blood cell deformability in sickle cell anaemia. Br J Haematol 168: 728-736, 2015.

24. Grau M, Pauly S, Ali J, Walpurgis K, Thevis M, Bloch W, and Suhr F. RBCNOS-dependent S-nitrosylation of cytoskeletal proteins improves RBC deformability. PloS one 8: e56759, 2013.

25. Gulliver G. Medical Times and Gazette. London: John Churchill \& Sons 1862.

26. Hertz L, Huisjes R, Llaudet-Planas E, Petkova-Kirova P, Makhro A, Danielczok JG, Egee S, Del Mar Manu-Pereira M, van Wijk R, Vives Corrons JL, Bogdanova A, and Kaestner L. Is Increased Intracellular Calcium in Red Blood Cells a Common Component in the Molecular Mechanism Causing Anemia? Frontiers in physiology 8: 673, 2017.

27. Horobin JT, Watanabe N, Hakozaki M, Sabapathy S, and Simmonds MJ. Shearstress mediated nitric oxide production within red blood cells: A dose-response. Clin Hemorheol Microcirc 2018.

28. Joiner CH. Gardos pathway to sickle cell therapies? Blood 111: 3918-3919, 2008.

29. Kaestner L. Channelizing the red blood cell: molecular biology competes with patchclamp. Front Mol Biosci 2: 46, 2015.

30. Kirklin JK, Pagani FD, Kormos RL, Stevenson LW, Blume ED, Myers SL, Miller MA, Baldwin JT, Young JB, and Naftel DC. Eighth annual INTERMACS report: Special focus on framing the impact of adverse events. J Heart Lung Transplant 36: 10801086, 2017.

31. Kleinbongard P, Schulz R, Rassaf T, Lauer T, Dejam A, Jax T, Kumara I, Gharini P, Kabanova S, Ozuyaman B, Schnurch HG, Godecke A, Weber AA, Robenek M, Robenek H, Bloch W, Rosen P, and Kelm M. Red blood cells express a functional endothelial nitric oxide synthase. Blood 107: 2943-2951, 2006. 
403 32. Kuck L, Grau M, Bloch W, and Simmonds MJ. Shear Stress Ameliorates 404 Superoxide Impairment to Erythrocyte Deformability With Concurrent Nitric Oxide Synthase 405 Activation. Front Physiol 10: 36, 2019.

406 33. Lauf PK, and Adragna NC. Properties and membrane transport mechanisms of 407 erythrocytes. In: Erythrocytes: Physiology and pathophysiologyWorld Scientific, 2012, p. 57 408228.

409 34. Lew VL, and Tiffert T. On the Mechanism of Human Red Blood Cell Longevity: 410 Roles of Calcium, the Sodium Pump, PIEZO1, and Gardos Channels. Frontiers in physiology 411 8: 977, 2017.

412 35. Lew VL, Tsien RY, Miner C, and Bookchin RM. Physiological [Ca2+]i level and 413 pump-leak turnover in intact red cells measured using an incorporated Ca chelator. Nature 414 298: 478-481, 1982.

415 36. Lux SEt. Anatomy of the red cell membrane skeleton: unanswered questions. Blood 416 127: 187-199, 2016.

417 37. Maher AD, and Kuchel PW. The Gardos channel: a review of the Ca2+-activated

$418 \mathrm{~K}+$ channel in human erythrocytes. The international journal of biochemistry \& cell biology 419 35: 1182-1197, 2003.

420 38. Mehta JL, Mehta P, and Li D. Nitric oxide synthase in adult red blood cells: vestige 421 of an earlier age or a biologically active enzyme? J Lab Clin Med 135: 430-431, 2000.

422 39. Nagarajan S, Raj RK, Saravanakumar V, Balaguru UM, Behera J, Rajendran 423 VK, Shathya Y, Ali BM, Sumantran V, and Chatterjee S. Mechanical perturbations 424 trigger endothelial nitric oxide synthase activity in human red blood cells. Sci Rep 6: 26935, 4252016.

426 40. Nguetse CN, Purington N, Ebel ER, Shakya B, Tetard M, Kremsner PG, 427 Velavan TP, and Egan ES. A common polymorphism in the mechanosensitive ion channel 428 PIEZO1 is associated with protection from severe malaria in humans. Proc Natl Acad Sci U S 429 A 117: 9074-9081, 2020.

430 41. O'Rear EA, Udden MM, McIntire LV, and Lynch EC. Reduced erythrocyte 431 deformability associated with calcium accumulation. Biochimica et biophysica acta 691: 274$432280,1982$.

433 42. Pasini EM, Kirkegaard M, Mortensen P, Lutz HU, Thomas AW, and Mann M. 434 In-depth analysis of the membrane and cytosolic proteome of red blood cells. Blood 108: 435 791-801, 2006.

436 43. Rooks H, Brewin J, Gardner K, Chakravorty S, Menzel S, Hannemann A, 437 Gibson J, and Rees DC. A gain of function variant in PIEZO1 (E756del) and sickle cell 438 disease. Haematologica 104: e91-e93, 2019.

439 44. Sanchez FA, Savalia NB, Duran RG, Lal BK, Boric MP, and Duran WN. 440 Functional significance of differential eNOS translocation. American journal of physiology 441 Heart and circulatory physiology 291: H1058-1064, 2006.

442 45. Shiga T, Sekiya M, Maeda N, Kon K, and Okazaki M. Cell age-dependent changes 443 in deformability and calcium accumulation of human erythrocytes. Biochimica et biophysica 444 acta 814: 289-299, 1985.

445 46. Shutova MS, and Svitkina TM. Common and Specific Functions of Nonmuscle 446 Myosin II Paralogs in Cells. Biochemistry (Mosc) 83: 1459-1468, 2018.

447 47. Simchon S, Jan KM, and Chien S. Influence of reduced red cell deformability on 448 regional blood flow. Am J Physiol 253: H898-903, 1987.

449 48. Simmonds MJ, Sabapathy S, Gass GC, Marshall-Gradisnik SM, Haseler LJ, 450 Christy RM, and Minahan CL. Heart rate variability is related to impaired haemorheology 451 in older women with type 2 diabetes. Clinical hemorheology and microcirculation 46: 57-68, 4522010. 
49. Smith AS, Nowak RB, Zhou S, Giannetto M, Gokhin DS, Papoin J, Ghiran IC,

454 Blanc L, Wan J, and Fowler VM. Myosin IIA interacts with the spectrin-actin membrane

455 skeleton to control red blood cell membrane curvature and deformability. Proc Natl Acad Sci

456 U S A 115: E4377-E4385, 2018.

457 50. Stocker JW, De Franceschi L, McNaughton-Smith GA, Corrocher R, Beuzard Y,

458 and Brugnara C. ICA-17043, a novel Gardos channel blocker, prevents sickled red blood 459 cell dehydration in vitro and in vivo in SAD mice. Blood 101: 2412-2418, 2003.

460 51. Suhr F, Brenig J, Muller R, Behrens H, Bloch W, and Grau M. Moderate exercise 461 promotes human RBC-NOS activity, NO production and deformability through Akt kinase 462 pathway. PloS one 7: e45982, 2012.

463 52. Takakuwa Y, Ishibashi T, and Mohandas N. Regulation of red cell membrane 464 deformability and stability by skeletal protein network. Biorheology 27: 357-365, 1990.

465 53. Tomaiuolo G. Biomechanical properties of red blood cells in health and disease 466 towards microfluidics. Biomicrofluidics 8: 051501, 2014.

467 54. Ulker P, Yaras N, Yalcin O, Celik-Ozenci C, Johnson PC, Meiselman HJ, and

468 Baskurt OK. Shear stress activation of nitric oxide synthase and increased nitric oxide levels 469 in human red blood cells. Nitric oxide : biology and chemistry 24: 184-191, 2011.

470 55. von Tempelhoff GF, Schelkunov O, Demirhan A, Tsikouras P, Rath W, Velten 471 E, and Csorba R. Correlation between blood rheological properties and red blood cell 472 indices( $\mathrm{MCH}, \mathrm{MCV}, \mathrm{MCHC})$ in healthy women. Clinical hemorheology and 473 microcirculation 62: 45-54, 2016.

474 56. Wang CH, and Popel AS. Effect of red blood cell shape on oxygen transport in 475 capillaries. Math Biosci 116: 89-110, 1993.

476 57. Weed RI, LaCelle PL, and Merrill EW. Metabolic dependence of red cell 477 deformability. J Clin Invest 48: 795-809, 1969.

478 58. Yoon C, Choi C, Stapleton S, Mirabella T, Howes C, Dong L, King J, Yang J, 479 Oberai A, Eyckmans J, and Chen CS. Myosin IIA-mediated forces regulate multicellular 480 integrity during vascular sprouting. Mol Biol Cell 30: 1974-1984, 2019. 


\section{FIGURE LEGENDS}

484 Figure 1: The physical properties classically known to facilitate the remarkable capacity of

485 RBC to reversibly deform.

486

Figure 2: The molecular composition of the cytoskeletal component in the RBC membrane, that resides under the lipid bilayer; $\alpha$ - and $\beta$-spectrins self-associate head-to-head, while each dimer is anchored to the lipid bilayer via either ankyrin or actin-4.1 complexes. Myosin IIA connects actin molecules within the junctional complexes and thus stabilises the bi-concave 491 shape of the RBC.

Figure 3: A synthesis of the observed regulatory pathways involving nitric oxide (NO) 494 generation and calcium ion-movement $\left(\mathrm{Ca}^{2+}\right)$ within $\mathrm{RBC}$ yields a complex intracellular signaling network. Mechanical stimulation via exposure to fluid shear in blood promotes $\mathrm{Ca}^{2+}$-influx via the mechanosensitive piezol-channel (1). Complexation of $\mathrm{Ca}^{2+}$ with the carrier protein calmodulin also occurs, which then collectively bind RBC-NOS (2). RBC-

498 NOS is subsequently activated, producing NO which appears to bind to $\alpha$ - and $\beta$-spectrins in 499 close proximity via S-nitrosylation of free cysteine residues, leading to increased flexibility 500 of the cytoskeleton and improved cellular deformability (3). Activation of the Gárdos channel

501 occurs in response to sustained $\mathrm{Ca}^{2+}$-influx (4), which facilitates export of potassium ions

$502\left(\mathrm{~K}^{+}\right)$and leads to a loss of intracellular fluid (5). Simultaneously, $\mathrm{Ca}^{2+}$ is slowly transported 503 out of the RBC via the plasma membrane $\mathrm{Ca}^{2+}$-ATPase (6). 
Cytosolic haemoglobin concentration dictates intracellular viscosity
Bi-concavity facilitates capacity to deform via high surface area to volume ratio

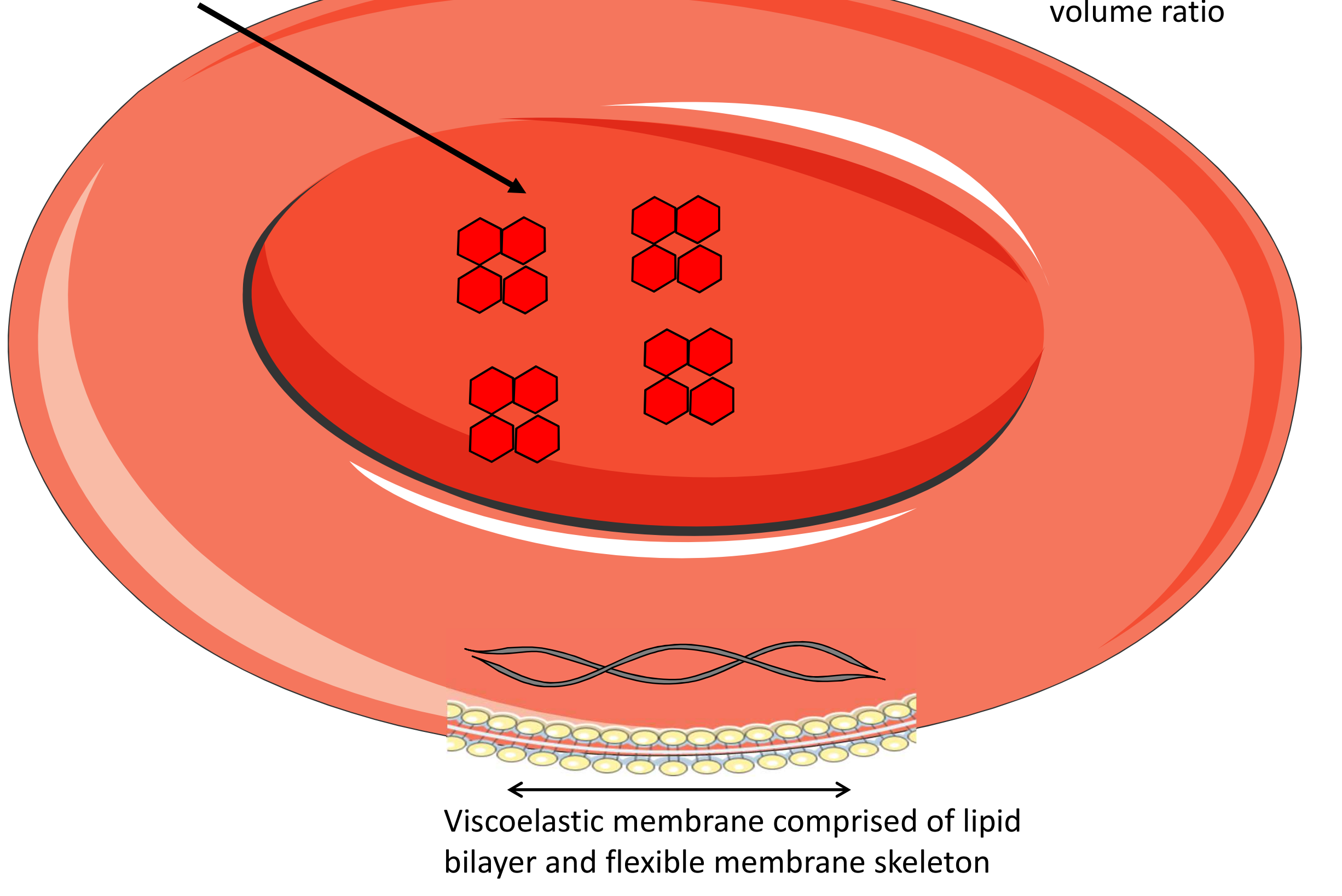




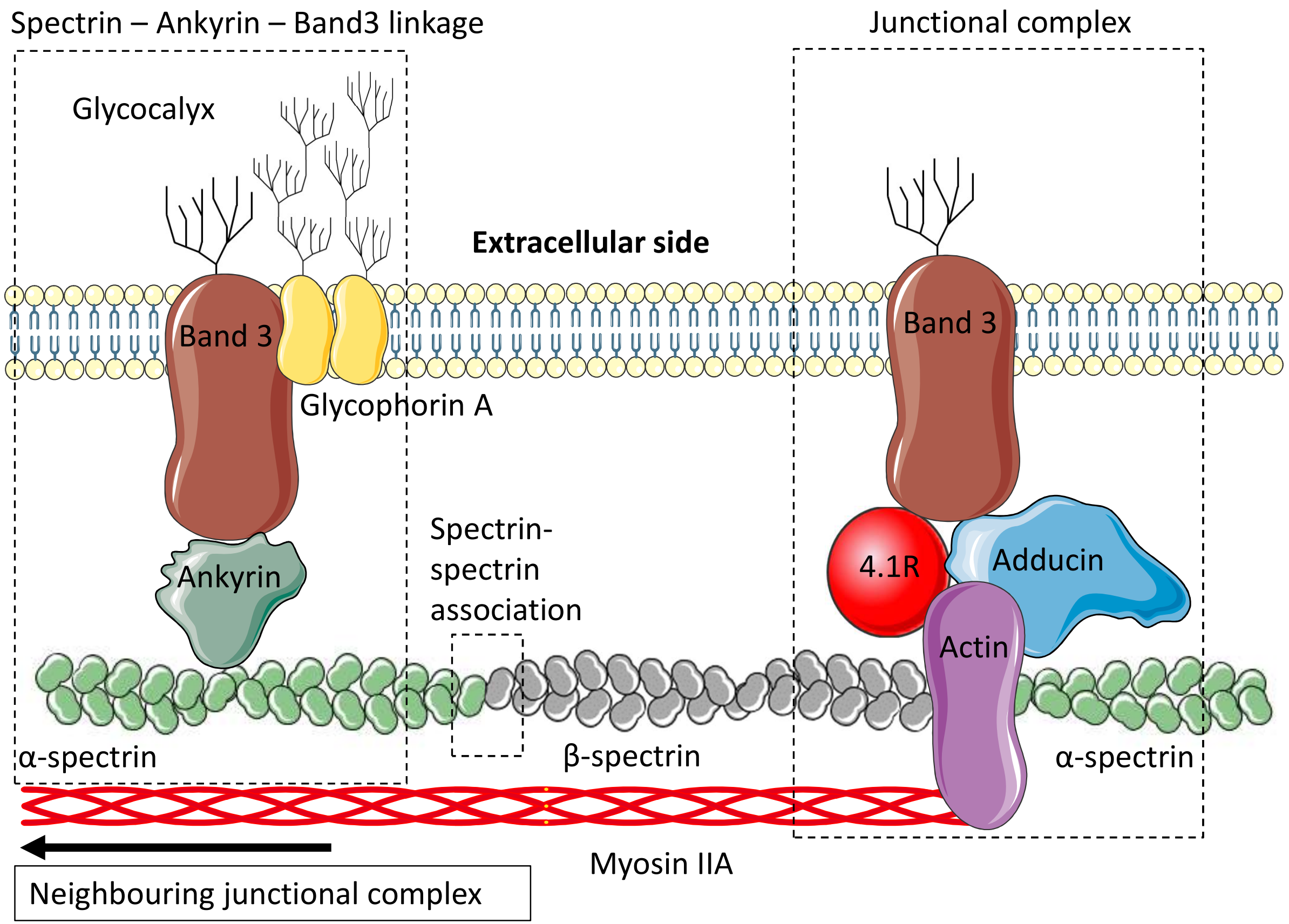

\section{Cytosol}




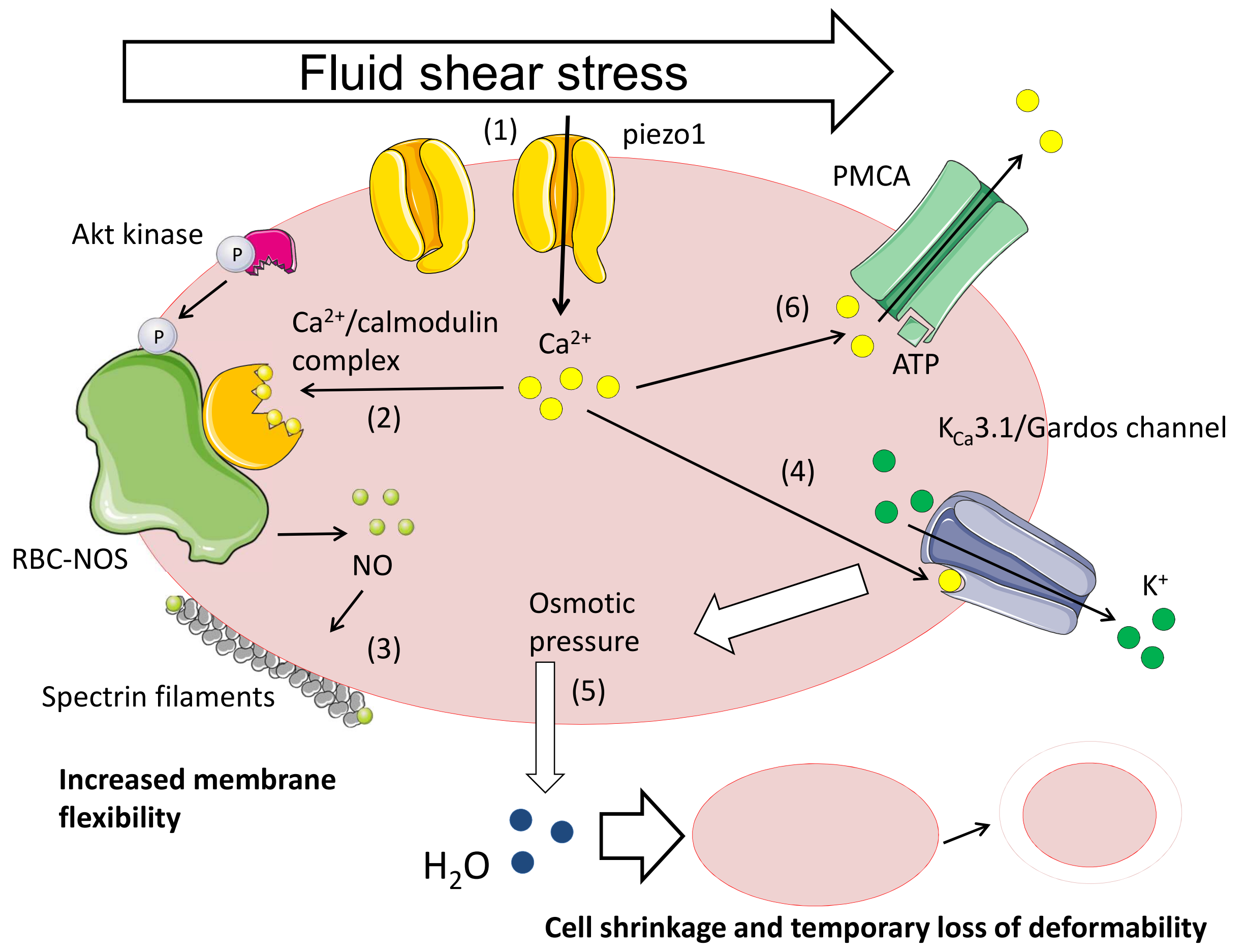

